\title{
Primary Pleural Rhabdomyosarcoma: Plain Film, CT and MRI Findings of This Extremely Rare Intrathoracic Tumor
}

\author{
Thomas Ray S. Sanchez ${ }^{1 *}$, Chirag V. Patel ${ }^{2}$, Noriko Satake ${ }^{2}$, Gary W. Raff ${ }^{2}$, Dariusz Borys ${ }^{2}$ \\ ${ }^{1}$ Departments of Radiology, Pediatrics, Surgery and Pathology, University of California Davis, Sacramento, USA \\ ${ }^{2}$ University of California Davis, Sacramento, USA \\ E-mail: "thomas.sanchez@ucdmc.ucdavis.edu \\ Received October 27, 2011; revised November 25, 2011; accepted December 5, 2011
}

\begin{abstract}
Primary pleural rhabdomyosarcoma is an extremely rare intrathoracic malignancy. We present a case of a previously healthy 2-year-old male complaining of cough and shortness of breath. The plain film, CT and MRI descriptions of this pleural tumor are presented. This is a fast growing tumor that is indistinguishable radiographically from other large intrathoracic tumors such as pleuropulmonary blastoma.
\end{abstract}

Keywords: Pleural Rhabdomyosarcoma, Intrathoracic, Pediatric, MRI

\section{Introduction}

Rhabdomyosarcoma is the most common malignant soft tissue tumor in the pediatric age group and usually arises in the head and neck region. An intrathoracic location is rare [1,2], with reported involvement of the lungs, bronchi, heart and mediastinum [3]. To our knowledge, only 5 cases of pleural rhabdomyosarcoma have been reported in the English literature. A single case report 20 years ago documented an intrathoracic mass, histology-proven to be a primary pleural rhabdomyosarcoma [4]. However, they failed to demonstrate a tumor aside from the $5 \mathrm{~mm}$ pleural thickening with associated pleural effusion and were not able to characterize the radiologic image of this lesion. Four more cases have been listed in the Intergroup Rhabdomyosarcoma Study (IRS) but without documentation of its imaging characteristics [5]. We present a case of a primary pleural embryonal rhabdomyosarcoma describing its characteristics on plain radiography, CT and MRI.

\section{Case Report}

A 2-year-old previously healthy male presented with 3 day history of cough and shortness of breath. Physical examination revealed intercostal retractions and absent breath sounds on the left. Chest X-ray (Figure 1) showed homogenous opacification of the left hemithorax with associated contralateral mediastinal shift. Empyema was initially suspected despite the absence of fever. However,

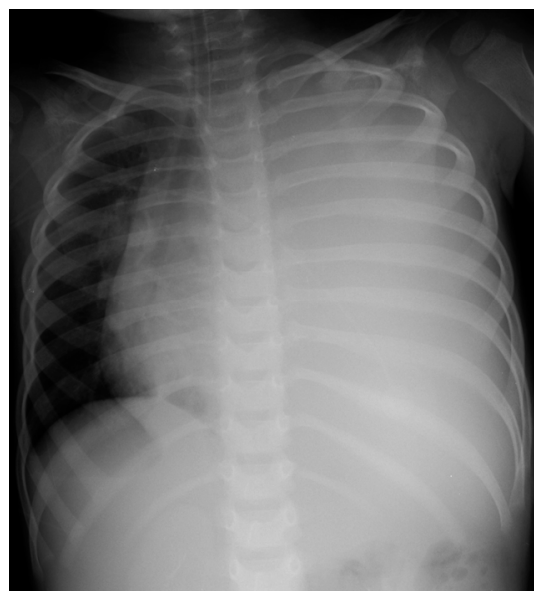

Figure 1. Chest radiograph demonstrated complete opacification of the left hemithorax initially suspected to be secondary to a large empyema.

CT of the chest (Figure 2) showed a large, heterogeneously enhancing left hemithorax mass. A preoperative chest MRI (Figure 3) was performed to evaluate possible mediastinal invasion. Images revealed a large solid mass occupying the entire left hemithorax with no mediastinal involvement or intraabdominal extension. Because of airway obstruction and failure of respiratory support, the patient underwent emergency resection of the tumor. Intraoperative findings revealed a large pleural based tumor, enveloped and derived its blood supply from the visceral and parietal pleurae. Tumor was partially adherent to a small portion of the pericardium and 


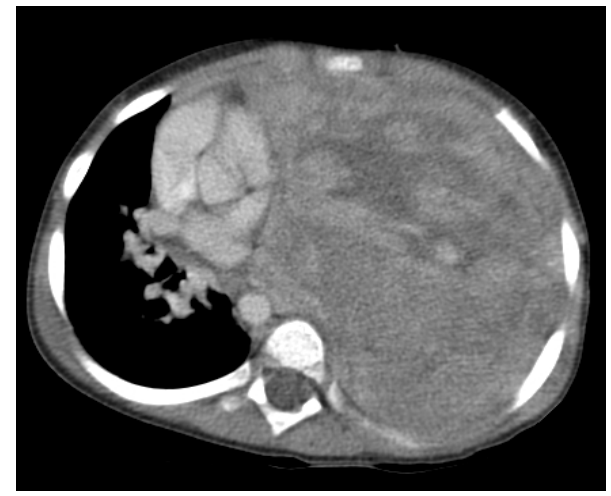

Figure 2. CT of the chest revealed a solid and heterogeneously enhancing mass filling the entire left chest with associated contralateral mediastinal shift.

segments of the left upper and lower lobes. These were also resected along with the tumor and submitted for histologic analysis. Findings on histologic sections and immunohistochemistry (Figure 4) showed proliferation of round to spindle cells with focal rhabdoid differentiation with strong positivity for myo-D in sarcoma cells all consistent with embryonal rhabdomyosarcoma. Resected pericardial and pulmonary components mainly showed inflammation and hemorrhage without evidence of malignant components. Post resection MRI (Figure 5(a)) showed residual enhancing apicopleural thickening compatible with tumor residual. Indeed the surgeon reported inability to completely remove the entire tumor due to the massive size. The CT scan showed post operative changes from partial resection of the pericardium and adjacent segment of the left lower lobe (Figure 5(b)). For the most part, the mediastinum, pericardium and chest wall appear intact supporting the surgical finding of a large mass arising from the pleural layers. The patient completed his chemotherapy and treated per Children's Oncology Group (COG) protocol ARST0431. It was decided that no radiation treatment was necessary. Follow up CT scan after 2 months showed resolution of the residual tumor in the left upper lobe. Two years after tumor resection and completion of chemotherapy, the patient is doing well and presently has no signs of tumor recurrence.

\section{Discussion}

Rhabdomyosarcoma is the most common soft tissue sarcoma in the first two decades of life, arising where skeletal or its mesenchymal anlage is present. It accounts for $10 \%$ of solid tumors in childhood and is the 3rd most common after neuroblastoma and Wilm's tumor [3]. Although the thoracic wall is involved in up to $70 \%$ of cases [6], pulmonary, mediastinal and pleural primaries are rare. The first reported case of a rhabdomyosarcoma arising from a pleural membrane documented thickening of the parietal pleura by computed tomography [4]. The MRI characteristic of this uncommon neoplasm has never been described in the literature.

Intrathoracic rhabdomyosarcoma is a fast growing,

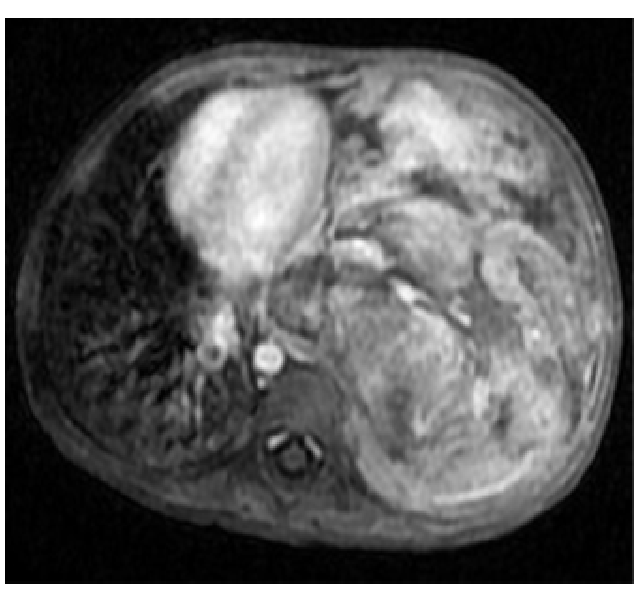

(a)

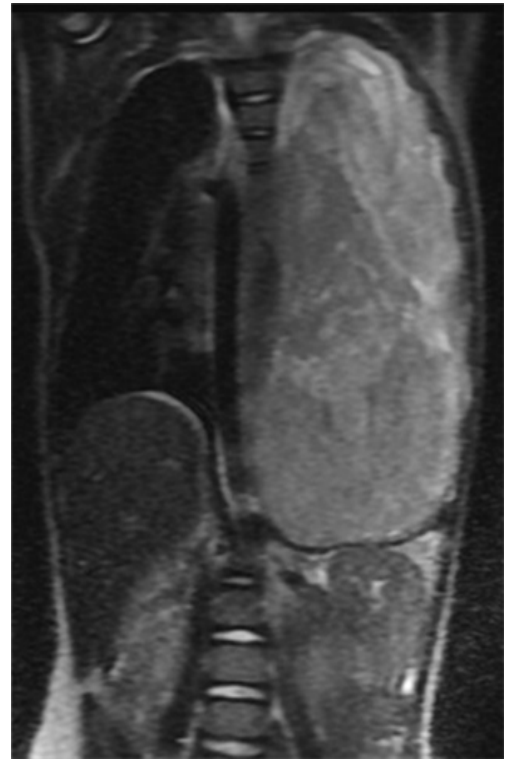

(b)

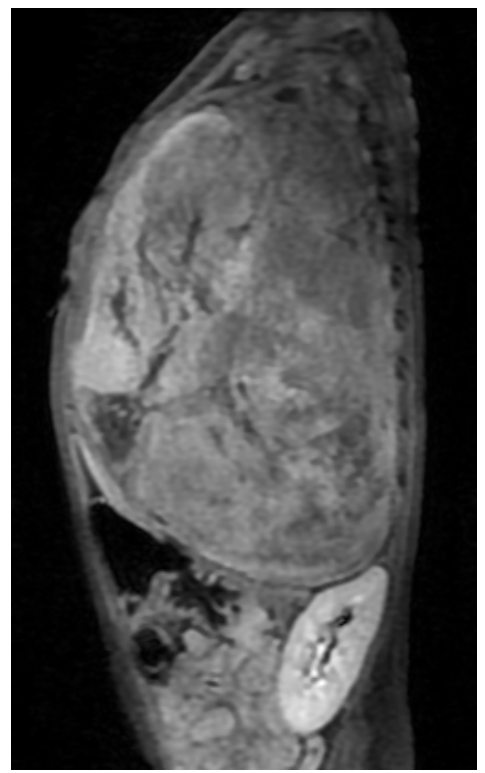

(c)

Figure 3. Axial, coronal and sagittal SSFSE T2 MRI again demonstrated the heterogeneous left hemithorax mass with no evidence of mediastinal involvement or intraabdominal extension. Despite the size of the mass, no significant areas of necrosis are present. 


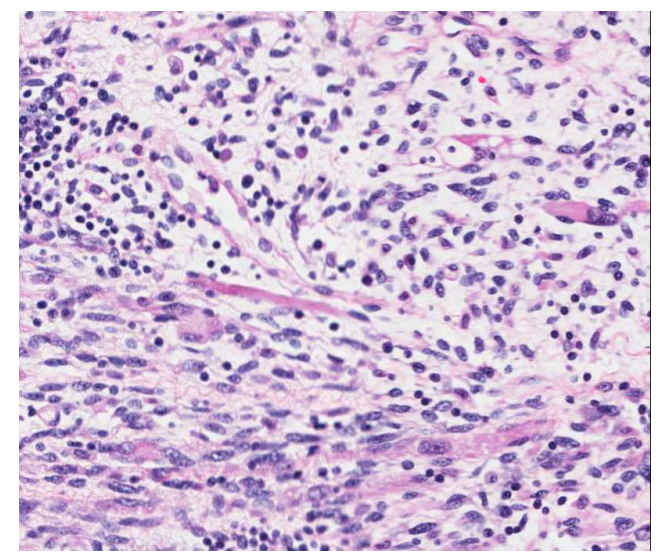

(a)

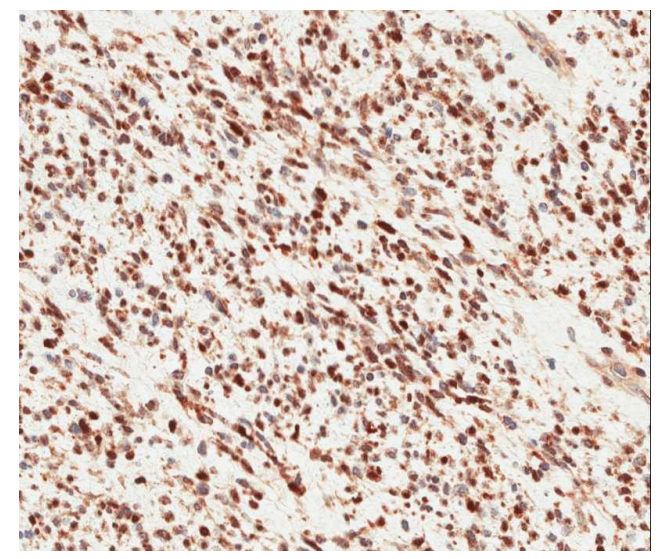

(b)

Figure 4. (a) High power view (20×) showed proliferation of round to spindle cells with focal rhabdoid differentiation with (b) strong positivity for myo-D in sarcoma cells.

usually painless mass which is large at presentation and associated with mediastinal shift or compression and sometimes invasion of the phrenic nerve [3]. This aggressive tumor can infiltrate the chest wall, mediastinum or extend outside the chest wall to involve the subcutaneous soft tissues [7]. Findings on plain radiographs of the chest can reveal abnormalities in the lung, mediastinum and pleura. The size of the tumor and related mass effect can also be seen but is insufficient in defining the entire extent of the neoplasm needed for proper surgical planning. CT scanning can adequately characterize the tumor extent as well as the presence of metastasis elsewhere. However, MRI with its multiplanar capability and greater soft tissue characterization is more sensitive in defining adjacent organ involvement or vascular invasion.

On MRI, the tumor appears isointense to muscle on T1-weighted images and intermediate to hyperintense on T2-weighted images. Heterogeneous but intense contrast enhancement is evident after intravenous administration

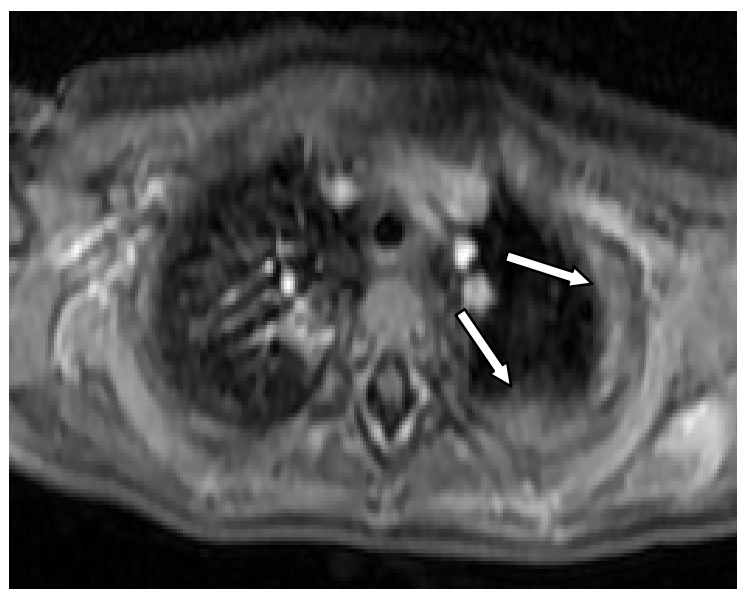

(a)

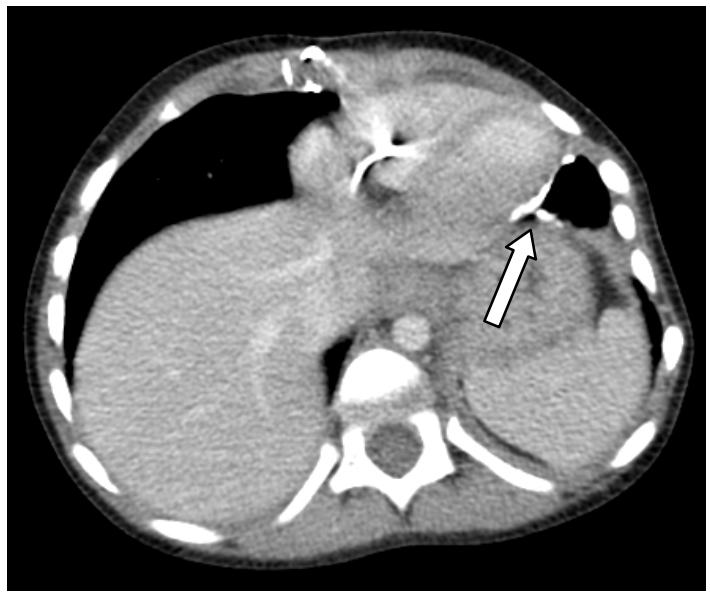

(b)

Figure 5. Post surgical imaging of the chest. (a) MRI revealed an enhancing residual left apicopleural thickeing (arrows) consistent with tumor residual; (b) Follow up CT scan after two months showed resolution of the residual pleural mass (not shown) and post surgical changes involving the partially resected pericardium and segment of the left lower lobe (arrow).

of Gadolinium (Gd-DTPA). Despite the massive size of the tumor in this patient, there are only minimal areas of necrosis and cystic degeneration indicated by the nonenhancing small hypointense foci centrally. This is probably due to significant tumor neovascularization as evidenced by prominent enhancing vessels within the mass and significant tumor neovascularization as seen intraoperatively. It has been reported that pleural effusion is rare in intrathoracic rhabdomyosarcomas [6]. However, their statistics did not include pleural primaries. Indeed, there is very limited experience with this extremely rare pleural malignancy and it is unsure whether the presence of an effusion is the rule. One would surmise that the tumor's pleural location would cause irritation or inflammatory reaction that would lead to at least a 
small amount of effusion. The case report by Hamada did present with pleural effusion. Our patient however did not.

Primary intrathoracic rhabdomyosarcoma is more aggressive than rhabdomyosarcomas arising from other sites $[3,6]$. It also carries a poor prognosis mainly due to its late presentation, advance stage and technical difficulty in local controls by resection or radiation $[5,8]$. Differential diagnosis for pediatric intrathoracic malignant tumors includes neuroblastoma, germinoma, lymphoma, pleuropulmonary blastoma and lung metastases which can be solitary and large [7]. Intrathoracic rhabdomyosarcomas are radiographically indistinguishable from solid pleuropulmonary blastoma (PPB). Both are rare and present as fast growing, large intrathoracic tumors in children. They are also both aggressive and prone to cerebral metastasis with low survival rates [2]. In conclusion, pleural rhabdomyosarcoma is a very rare intrathoracic malignancy that can be considered in a young patient presenting with a large and solid mass. MRI is the most sensitive tool in evaluating tumor extension crucial for surgical planning.

\section{References}

[1] N. Martini, S. Hajdu and E. Beattie, "Primary Sarcoma of the Lung," The Journal of Thoracic and Cardiovascular Surgery, Vol. 61, No. 1, 1971, pp. 33-38.

[2] M. Cohen and R. O. C. Kaschula, "Primary Pulmonary Tumors in Childhood: A Review of 31 Years' Experience and the Literature," Pediatric Pulmonology, Vol. 14, No. 4, 1992, pp. 222-232. doi:10.1002/ppul.1950140405
[3] V. G. McDermott, S. Mackenzie and G. M. Hendry, “Case Report: Primary Intrathoracic Rhabdomyosarcoma: A Rare Childhood Malignancy," British Journal of Radiology, Vol. 66, No. 790, 1993, pp. 937-941. doi:10.1259/0007-1285-66-790-937

[4] T. Hamada, A. Tanimoto, M. Kaido, S. Matsumoto, K. Iribe and O. Koide, "Diffuse Pleural Rhabdomyosarcoma with Persistent Pleural Effusion,” Acta Pathologica Japonica, Vol. 39, No. 12, 1989, pp. 803-809.

[5] R. J. Andrassy, E. S. Wiener, R. B. Raney, W. Lawrenece, T. E. Lobe, C. A. Corpron and H. M. Maurer, "Thoracic Sarcomas in Children,” Annals of Surgery, Vol. 227, No. 2, 1998, pp. 170-173. doi:10.1097/00000658-199802000-00003

[6] I. Cohen, N. Loberant, E. King, M. Herskovits, Y. Sweed and J. Jerushalmi, "Rhabdomyosarcoma in a Child with Massive Pleural Effusion: Cytological Diagnosis from Pleural Fluid,” Diagnostic Cytopathology, Vol. 21, No. 2, 1999, pp. 125-128. doi:10.1002/(SICI)1097-0339(199908)21:2<125::AID-D C9>3.0.CO;2-Z

[7] C. S. Schmaltz, S. Sauter, O. Opitz, D. Harms, B. Kremens, M. Lohner, K. Metz, M. Brandis and C. Niemeyer, "Pleuro-Pulmonary Blastoma: A Case Report and Review of the Literature,” Medical and Pediatric Oncology, Vol. 25, No. 6, 1995, pp. 479-484. doi:10.1002/mpo.2950250612

[8] W. M. Crist, R. B. Raney, W. Newton, W. Lawrence Jr., M. Tefft and M. A. Foulkes, "Intrathoracic Soft Tissue Sarcomas in Children,” Cancer, Vol. 50, No. 3, 1982, pp. 598-604. doi:10.1002/1097-0142(19820801)50:3<598::AID-CNC R2820500336>3.0.CO;2-\# 